\title{
AML-Erhaltungstherapie: Cytarabin intensivieren oder Anthrazyklin dazu?
}

\author{
Der Frage nach der optimalen \\ Konsolidierung bei akuter \\ myeloischer Leukämie (AML) gingen \\ koreanische Hämatologen nach.
}

$\mathrm{D}$ ie Standardtherapie der AML besteht aus einer anthrazyklinbasierten Induktion, gefolgt von einer Konsolidierung in Form einer Cytarabin-basierten Chemotherapie oder Stammzelltransplantation. Inzwischen versucht man durch eine geringere Dosierung von Cytarabin in der Konsolidierung die Toxizitäten zu reduzieren und durch Zugabe anderer Substanzen die Wirksamkeit $\mathrm{zu}$ steigern. In einer retrospektiven Auswertung wurde bei 145 AML-Patienten aus dem Register der Korea Universität, die durch die Induktion (3 + 7-Regime) eine vollständige Remission erreicht hat- ten, hoch dosiertes Cytarabin $\left(3 \mathrm{~g} / \mathrm{m}^{2}\right)$ mit niedriger dosiertem $\left(1 \mathrm{~g} / \mathrm{m}^{2}\right)$ in Kombination mit einem Anthrazyklin (Idarubicin oder Mitoxantron) verglichen. Bei einem Teil der Patienten erfolgte nach der Konsolidierung noch eine Stammzelltransplantation.

Nach median 16 Monaten war das Gesamtüberleben in der Hochdosis-Gruppe signifikant länger als in der Kombinationsgruppe (Median nicht erreicht vs. 16,6 Monate; 5-Jahres-Überlebensrate $55,4$ vs. $39,8 \%$; $\mathrm{p}=0,045)$. Multivariate Analysen ergaben, dass Performancestatus und Stammzelltransplantation unabhängige Prognosefaktoren waren. Allerdings war multivariat berechnet der Überlebensvorteil unter hoch dosiertem Cytarabin nicht mehr so deutlich ausgeprägt (Hazard Ratio 0,622; $\mathrm{p}=0,077$ ).
Auch das rezidivfreie Überleben war in der Hochdosis-Gruppe signifikant länger (median 38,6 vs. 11,0 Monate; $\mathrm{p}=0,011)$. Darüber hinaus dauerte eine Neutropenie unter der Kombinationstherapie länger als unter Hochdosis- $\mathrm{Cy}$ tarabin (median 10 vs. 8 Tage; $\mathrm{p}=0,001$ ). Die therapiebedingte Mortalität war in beiden Gruppen vergleichbar.

Fazit: Die Studie zeigt, dass eine Konsolidierung mit Hochdosis-Cytarabin eine bessere Prognose bietet als die Kombination aus Cytarabin in mittlerer Dosierung plus Anthrazyklin. Judith Neumaier

Kim DS et al. Comparison of consolidation strategies in acute myeloid leukemia: high-dose cytarabine alone versus intermediate-dose cytarabine combined with anthracyclines. Ann Hematol. 2015;94(9):1485-92.

\section{Sekundäre und therapiebedingte AML im Fokus}

\section{Sekundäre und therapiebedingte akute myeloische Leukämien (AML)} unterscheiden sich von einer De-novo-AML biologisch sowie prognostisch. Details wurden in einer populationsbasierten dänischen Studie untersucht.

Cekundäre und therapiebedingte AML (sAML bzw. tAML) bleiben eine therapeutische Herausforderung. Noch immer ist unklar, ob die im Vergleich zur De-novo-AML schlechtere Prognose durch die vorherige hämatologische Erkrankung oder durch Unter- schiede in den Karyotypen und/oder im Alter erklärt werden kann.

Häufigkeit (Abb. 1) und Eigenschaften von tAML, sAML nach myelodysplastischem Syndrom (MDS-sAML) und Nicht-MDS-sAML (chronische myelomonozytische Leukämie und myelopro-

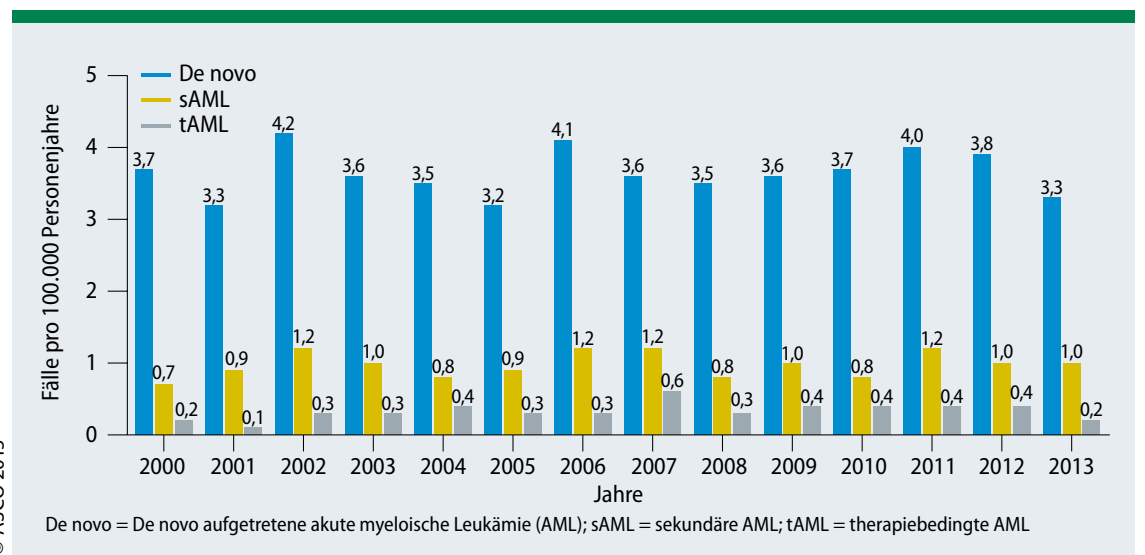

Abb. 1: Elne De-novo-AML war in allen ausgewerteten Jahren am häufigsten. 